This is an Accepted Manuscript of an article published by Taylor \& Francis in Journal of Maps on 22 February 2013 (date of online availability) available online:

http://wwww.tandfonline.com/10.1080/17445647.2013.773568 


\title{
Bibliographic webmap: the Physical Landscape of Britain and Northern Ireland
}

Piccinini, C. ${ }^{1}$, Smith, M.J. ${ }^{1 *}$, Hooke, J. ${ }^{2}$ and Hesketh, K. ${ }^{2}$

${ }^{1}$ School of Geography, Geology and the Environment, Kingston University

${ }^{2}$ Department of Geography, University of Liverpool

*michael.smith@kingston.ac.uk

\begin{abstract}
In this article we present the development of a web mapping application as part of the "Physical Landscape of Britain and Northern Ireland" project. There are a large number of sources for information and data on the geomorphology of the British landscape including academic journals, books, unpublished student dissertations and third party reports, however there remains no single central repository to query the extent of such knowledge. This project, sponsored by the British Society for Geomorphology, has a long term aim of collating a bibliography on the full extent of published and unpublished research on the geomorphology of Britain and Northern Ireland, ultimately highlighting "gaps" in our knowledge and identifying topics and areas for future investigation. The first phase of the project involved the development of a web application that allows end-users to search for bibliographic references using an interactive map. Here we outline the implementation of a spatial database, a database front-end and a web mapping application built using open source software and open data. Data for pilot areas has been entered; a further phase of the project will populate the database for other areas.
\end{abstract}

Keywords: geomorphology; map; web; UK. 


\section{Introduction}

Geomorphology is the science that analyses how climatic, tectonic and biogenic processes act on the surface of the Earth to create landforms and landscapes. Whilst many organisations recognise the importance of geomorphic processes and landforms in their work (e.g., engineering geology for large-scale infrastructure projects), there is no single centralised resource on the geomorphology of Britain. Some useful sources exist but these tend to be thematic (e.g., BRITICE (Clark, 2011)), spatially restricted (e.g., GeoEast (2011)) or broad-brush (e.g., National Character Areas (Natural England, 2011)). Moreover there are data sources such as government reports and PhD dissertations containing valuable information but of limited accessibility; the so-called "grey" literature.

The "Physical Landscape of Britain and Northern Ireland" project, organised as a working group of the British Society for Geomorphology, aims to enable the end-user to quickly and accurately ascertain the nature, and review the role, of geomorphological processes in shaping the physical landscape at any given location within Britain and Northern Ireland. More generically, it is to provide the professional, academic and general public with access to information, data and knowledge on the geomorphology of the British landscape, with information accessible through an interactive web mapping application built using both open data and open source technologies. The project is intended to provide an "umbrella" to understanding the breadth and depth of the geomorphology, and geomorphological research, in Britain and Northern Ireland. There are three primary stages to the over-arching project: (1) develop a web mapping application (and accompanying spatial database) with bibliographic data; (2) synthesise and interpret literature identified in (1) and (3) commission research to generate new data and understanding for regions where information is lacking.

This paper describes the technical implementation of the spatial database and web mapping application (http://www.landscapebritain.org.uk) which has been funded under a Natural Environment Research Council (NERC) Knowledge Exchange programme in partnership with Natural England, the Environment Agency, Mouchel, Halcrow and the National Trust.

\section{Methods}

In order to develop a web mapping application it was necessary to select an appropriate approach and subsequent underlying technology. Interactive web maps are an ideal platform to distribute information to a wide audience (e.g., Roth, et al, 2009; Brown and Kraak, 2001; Mitchell, 2008; Reuschel and Hurni, 2011), with end-users potentially varying from specialist to non-specialist. The site would need to function around core internet standards, be able to perform complex spatial queries, remain extensible and ideally use open source technologies (in order to allow license-free deployment). An underlying spatial database was therefore specified for spatial processing, with the majority of additional functionality performed in the user's web browser. Detailed base mapping was required for Britain and Northern Ireland; it was not feasible to source this data within the project constraints and therefore open access data from third parties was required. The 
application is therefore dependent upon the availability and specified cartography of third party basemap data (e.g., Bing Maps and OpenStreetMap), however the user can switch between these products within the interface and, given the widespread use of these basemaps, their interfaces will be familiar to users.

The above specification led to development based upon a Linux operating system running an Apache web server and PostgreSQL/PostGIS (see \$2.1) spatial database. PostgreSQL was selected due to interoperability with geographic information system (GIS) desktop software and web mapping libraries, spatial functions, general functions and usability (IT Management and Leadership, 2012; Westra, 2010). In particular PostgreSQL has good support for spatial functions, output formats and data loading/editing.

\subsection{Design Criteria}

The simplified architecture specified above means the system is portable, avoiding the complexity of the two main alternative approaches: Flash web map and server GIS. The Flash approach requires the use of a proprietary plugin that is not available on all operating systems, whilst a server GIS would require greater server specification and potentially lead to poorer application performance. One of the main advantages of the approach adopted is speed: there is no server GIS between the client and the database. The web browser (or client) sends messages directly to the web server as 'POST' or 'GET' HTTP requests, with the Smarty PHP Template engine is used to generate the GeoJSON file for map visualization (Obe and Hsu, 2011). Additional textual data to drive the client user interface is sent as simple JSON files (Figure 1). One drawback of this design is that it is not possible to use common web service standards such as Web Map Services (WMS) and Web Feature Services (WFS) to share data.

\subsection{Development}

Application development involved three stages: (i) database schema definition; (ii) web-based database management application and (iii) web-map development.

(i) Database Schema Definition: work began with the definition of a database schema. One of the requirements of the project is the storage of a location for each bibliographic reference which allows it to be spatially located on a map; a spatial database was therefore used.

(ii) Web-based Database Management: to ease database management by a data manager, an online bespoke front-end user interface was developed. There are different graphical user interfaces (GUIs) available to manage databases but they can be complex to use, moreover they do not allow the insertion of spatial features by drawing them. Instead the customised front-end interface is tailored to this project database schema which makes it easy to upload new data or edit existing data. The front-end hides the schema from the data manager thus avoiding some dangerous operations that could cause the database to become unusable. 
(iii) Web-map Application: a web mapping application has been built to access and visualize data on a client web browser. Both the front-end and the web mapping application use two JavaScript libraries available under an open source licence: ExtJS (http://www.sencha.com/) and OpenLayers (http://www.openlayers.org/). The Extjs 4.1 JavaScript framework is used to build the user interface and drive the application logic whilst the OpenLayers 2.11 JavaScript API is used to visualize spatial data. ExtJS was chosen because of the large number of user interface widgets and cross browser compatibility. OpenLayers is a library for building advanced mapping applications, allowing the use of spatial data stored in different formats and complex mapping visualizations. Figure 2 shows the general system architecture where the data manager uses the front-end to manage the database and the general user accesses the data using a client web browser.

The mapping application is targeted at both specialist and non-specialist users and allows the identification of bibliographic references on a base map. Searching for bibliographic references is carried out using both spatial and aspatial queries. With a simple query a non-specialist user can have an overview of bibliographic reference locations within a particular area of interest whilst a specialist user can deepen the search by carrying out a more complex query. Moreover a reporting system is included allowing the user to download results in different data formats, including Portable Document Format (PDF), Endnote eXtensible Markup Language (XML), comma separate values (CSV), GeoJSON (JavaScript Object Notation) and Keyhole Markup Language (KML).

Each reference location is stored in the spatial database as a bounding box. This simple shape has been chosen to ease data management and allow fast visualization. By default only the bounding boxes' centroids, not the rectangles, are displayed on the map to avoid visual clutter. Furthermore, a server-side clustering system groups the centroids as the user zooms out from the map. Clustering data allows the visualization of a large number of points while the user explores the map at a variety of scales.

\section{Architecture Implementation}

\subsection{Database}

The database schema was designed to maintain data integrity, whilst meeting user requirements for real-world use, extensibility and meeting statutory requirements for data transfer. Specifically the database must store primary bibliographic reference information (e.g., author, year, title, page numbers), with each reference classified by source type (e.g., book, journal article). Each reference has a location, with the location geometry stored as one or more bounding boxes. Additional classification or "tagging" allows more detailed consideration of the reference content and therefore greater granularity in database querying by end-users. A reference can also have an abstract and non-textual data such as imagery. The database must additionally store administrative boundaries to allow spatial queries and allow interaction with standardised international classification systems. 
The database entity-relationship (ER) schema is depicted in Figure 3, showing the actual database structure and relationships between tables. There are six main groups:

(1) 'Bibliographic' (red): contains the table 'ARTICLE' storing the main bibliographic reference information. Each reference is classified according to its' source type. Source types are stored in the 'SOURCE' table. At present possible sources are book, journal, dissertation, book chapter and unpublished report. Each reference can have an abstract stored in the 'ABSTRACT' table. Both the 'ABSTRACT' and the 'ARTICLE' tables implement full text searching used to pre-process text data and create an index for later rapid searching. A stored procedure automatically creates a new index each time text is added to the table. This allows using one or more keywords to filter abstracts and titles whilst searching for references.

(2) 'Multimedia' (yellow): these tables store optional images and static maps which can be viewed by the end user once a reference is selected.

(3) 'Spatia'l (green): the reference location is stored in the 'Spatial' group. A generic location is possible with the tables 'PLACE' and 'NCA'. The 'PLACE' table holds the Ordnance Survey Open Data Gazetteer (Ordnance Survey, 2012) at 1:50,000 scale containing around 260,000 points for Great Britain; if necessary additional gazetteers can be added at a later stage. The 'NCA' table stores the 159 areas that subdivide England into areas of similar landscape character, National Character Areas. Similar to the 'PLACE' table, additional landscape classifications can be added for Wales, Scotland and Northern Ireland at a later stage. Each reference is assigned a main place and main NCA; a more detailed location is possible with one or more bounding boxes stored in the 'LOCATION' table.

(4) 'Geographical features' (yellow): this group contains several spatially enabled tables storing various kinds of boundaries. Specifically the following tables are available: Civil Administration Areas, National Natural Reserves, RAMSAR sites, Sites of Special Scientific Interest, Special Areas of Conservation and Special Protection Areas. These geometries are used to search for references using spatial queries.

(5) 'Level' (orange): various key terms ("tags") can be used to classify a reference. Here we implemented a hierarchical 3-level classification. The 'Level' group contains the three tables holding the key terms for each level. Each reference is classified with one of the nine key terms belonging to the level 1: environment/landform, processes, impact, material, timescale or period, attribute within system, hazards, technique, and management. Level 2 key terms further classify a level 1 key term. For some level 2 terms additional level 3 terms are available (Table 1).

(6) 'INSPIRE' (blue): contains the tables 'NaturalGeomorphologicFeatureType' and 'AnthropogenicGeomorphologicFeatureType' which hold a classification system based on the draft INSPIRE (I nfrastructure for Spatial Information in Europe) data specification on geology D2.8.II.4_v2.0.1. (INSPIRE, 2011). They are linked to the 'LEVEL2' and 'LEVEL3' tables thus 
allowing a relationship between this project classification and the European INSPIRE classification. This makes it possible to search for references using both classification systems.

\subsection{Database front-end}

Application development included an administrative "front-end" to ease database management, with a particular focus upon bibliographic reference creation, editing and deletion. The front-end allows the insertion of all the data necessary to define a bibliographic reference: it is possible to insert an abstract, classify a reference using the 3-level classification system, upload images and maps and create the reference bounding box using an interactive map (Figure 4 and Video 1 ).

In addition, the bulk-upload of references from Endnote (using an XML file format) is supported; therefore the data manager can collect a large group of references and abstracts and upload them as a batch job to the database, although this requires subsequent manual geocoding.

\subsection{Web Mapping Application}

In designing the web mapping application GUI, the challenge was to present the interactive map as the centrepiece of the design whilst also allowing sophisticated methods of database query and reporting. The project partners were the primary target audience, predominantly professional users and academic researchers. The GUI design comprises four main parts: a map panel, a vertical panel, a horizontal panel and a reference details panel (Figure 5). However users can customize the GUI to suit their own preferences: to free up more space for the map it is possible to close both the vertical and horizontal panels, panels can also be detached from the main GUI.

The map panel within the web application contains two elements: a background map and vector layers which are overlain to depict the locations of bibliographic information. OpenStreetMap data is loaded as the default basemap (given the requirment to utilise third party data), although users can opt to choose other basemaps, including Bing topographic maps and aerial imagery. Other vector layers will become available for rendering as the project progresses, with this currently limited to National Character Areas; users can change the layer symbology to better render it upon the selected basemap.

Once the database has been queried (see below) it is necessary to visualise the results; given the potentially large number of data points, all references are represented as a single opaque point in order to avoid visual clutter. When clicked, the reference bounding box (or boxes) is displayed with $50 \%$ opacity so that it can be viewed in reference to the underlying basemap. If the user zooms out there is again the possibility of visual clutter so a server-side clustering algorithm groups the centroids, indicating numerically the number of references in each cluster (see below).

The vertical panel contains tabs that allow access to different levels of functionality including "About" information, help, map legend, querying and basemap layers. The query tab allows querying of the database using both spatial and aspatial queries. Spatial queries are used to select 
references with bounding boxes that intersect the current map extent, intersect other features such as administrative boundaries or intersect a custom feature (point, line or polygon). Custom features can be interactively drawn. A buffer can be applied to both boundaries and custom features. Additional aspatial query parameters include the 3-level classification, reference attributes and abstract keywords.

When performing a spatial query a PHP script on the server receives parameters from the client web browser and creates a query string in a modular fashion (Figure 6). A spatial filter retrieves the IDs of references for which bounding boxes intersect the area of interest. An area can be the current client browser map extent, a feature boundary or a custom feature drawn on the client map. The IDs are then further filtered using optional key terms, reference attributes and abstract keywords. The resulting query string is used to retrieve tabular data which is then displayed in the client web browser.

In addition to displaying tabular data, spatial data is used to identify the location of the reference on the webmap. This involves a further step which filters the geometry shown on the map as either bounding box centroids or clusters. At small scales, features overlap and the map becomes cluttered; features are therefore displayed as clusters. Clustering is possible on the client browser but only for small numbers of features. Clustering hundreds of features is not feasible as the performance of the client GUI rapidly degrades. For this reason, at small scales data is clustered using database queries on the server before the results are sent to the client. Moreover, clustering displays fewer points on the client web browser further improving client application performance. Clustering only happens at small scales; at large scales there is no clustering, however only the centroids of the bonding boxes intersecting the client map extent are sent to the client. This further improves client application performance. When the user selects a cluster or centroid on the map, the corresponding row is highlighted in the table (and vice versa).

Spatial and geometry filtering always occur on the server; other more complex aspatial filters are dependent upon the queries built by the end user. These can become quite complex and in these situations it is useful to save the query parameters. This is implemented in the web application using HTML5 localstorage (w3schools, 2012), allowing the retrieval of up to 5 queries. After the user closes the web browser, the query parameters will be available the next time the browser is opened (as long as the web browser cache is not cleared). Localstorage availability is performed as the application starts; if the browser does not support localstorage this functionality is not available, however the user is able to copy and paste the query parameters as plain text.

Query results are presented in both tabular form (as reference data) and their locations are shown on the web map. Additional information such as charts and images are available for each selected reference and the user is able to download custom reports containing reference data.

\section{Discussion}


During development the mapping application was tested by a group of ten individuals comprised of professional users from the project partners (Natural England, the Environment Agency, Mouchel, Halcrow and the National Trust). Unstructured feedback via email was useful to identify and subsequently implement, usability and feature-set issues prior to the release of the first version. In general the users appreciated both the proposed user interface and functions with only a few changes necessary. For example, changing the position of several icons and adding tooltips to all the buttons were identified in order to improve usability. Additional interaction between the application and the end user was added, for example by means of information messages. We added the EndNote XML file as one of the formats to download a single reference or a group of references making it easier for the user to change the formatting style as they prefer; we chose EndNote XML as this is one of the more commonly used file formats for managing bibliographic references. Online documentation, available through the help tab within the user interface, was added to help users interact with the application particularly as it is targeted at academics, professionals and the general public.

Some users found it confusing that a result can have points falling outside the search area. This happens because the map displays a reference bounding box centroid, but the spatial query outcome is obtained by intersecting a search area with the reference bounding boxes. It is not uncommon for the centroid of the bounding box to fall outside the feature boundary, particularly if the bounding box has an elongated rectangular shape. A reference can also have multiple bounding boxes, therefore a query will return all the reference bounding boxes including those that are not intersecting the area of interest. Visualizing the bounding boxes would have made the map confusing as many of them can overlap. Therefore only the centroids are shown; the bounding boxes appear when the user selects the centroid. Using the centroids also makes it easier to set up a clustering algorithm on the database. Another source of confusion was the automatic selection of multiple points and visualization of multiple bounding boxes when a single point is selected. Again, this is due to the fact that a reference can have more than one bounding box and selecting one of them selects all the others because they all belong to the same reference. With the launch of the application in December 2012, an online survey with structured closed and openended questions will query users on both their intended use of the system and their experiences in its usability.

The web application (and source code) is available at http://www.landscapebritain.org.uk. Future development includes adding new functions such as: (1) 3D visualization (using the OpenWebGlobeSDK ) and (2) a simplified mobile version (using Touch2 SDK). 3D visualisation of a landscape where active processes operate, could provide valuable contextual information for users spatially browsing the bibliographic database. Likewise, mobile devices can potentially add value to the application if they have a built-in global positioning system receiver, thus allowing the user's position to perform spatial queries and thereby identify potential relevant literature as a result of their location. 


\section{Conclusions}

This project has focused upon the development of a spatial database and accompanying database administration and web mapping applications for the provision of reference information on the geomorphology of the British landscape to professional end-users and the general public. Initial focus has been on the compilation of reference information for two areas, including part of the High Speed 2 (HS2) rail link between London and the West Midlands. Reference sources at this stage include published journal articles and books.

The client front-end allows easy administration of records in the underlying database, including the bulk import of references from Endnote XML files. Additionally, a bespoke mapping tool allows the insertion and modification of bounding boxes together with other table attributes. A 3level classification system allows category "tagging", aiding end-users in database searches. On completion in December 2012, the project concluded technical development and the compilation of reference information, in detail, for two sample areas. Future work will look to compile full reference information for Britain and Northern Ireland, further helping to identify gaps in knowledge and help direct future geomorphological research, however this is subject to further funding.

\section{Acknowledgements}

We gratefully acknowledge the support of the Natural Environment Research Council Knowledge Exchange Grant (NE/J00636X/1).

\section{Software}

PostgreSQL with PostGIS extensions is used to store all spatial and aspatial data using a Linux server. The Apache web server with PHP is used for all server side scripting. Extjs 4.1 and OpenLayers 2.11 are used on the client-side web browser for all user interaction.

\section{References}

Brown, A., Kraak, M.-J., 2000. Web Cartography. CRC Press, London.

Clark, C.D., 2011. BRITICE - The British Ice Sheet. Accessed: 7 November 2011. http://www.sheffield.ac.uk/geography/staff/clark_chris/britice.

GeoEast, 2011. Places to visit. Publicly accessible geodiversity sites and features in the East of England. Accessed: 7 July 2011. http://www.geo-east.org.uk/placestovisit.htm.

INSPIRE, 2011. D2.8.II.4 Data Specification on Geology - Draft Guidelines. Accessed: 7 May 2012. http://inspire.jrc.ec.europa.eu/documents/Data Specifications/INSPIRE DataSpecification GE v2. 01.pdf. 
IT Management and Leadership eMagazine, 2012. PostgreSQL vs. MySQL, Pros \& Cons. Accessed: 7 May 2012. http://leadit.us/hands-on-tech/PostgreSQL-vs-MySQL-Pros-Cons.

Mitchell, T., 2008. Web Mapping Illustrated: Using Open Source GIS Toolkits. O'Reilly Media.

Natural England, 2012. Search for a National Character Area. Accessed:7 November 2011.

http://www.naturalengland.org.uk/publications/nca/searchpage.aspx.

Obe, R., Hsu, L., 2011. PostGIS In Action. Manning, Stamford.

Ordnance Survey, 2012. OS OpenData. Accessed: 4 May 2011.

http://www.ordnancesurvey.co.uk/oswebsite/products/os-opendata.html.

Reuschel, A-K., and Hurni, L., 2011. Mapping literature: visualisation of spatial uncertainty in fiction. The Cartographic Journal, 48, 4, 293-308.

Roth, R.E., Van Den Hoek, J., Woodruff, A.W., Erkenswick, A., McGlynn, E., Przybylowski, J., 2009. The 21st Century Campus Map: Mapping the University of Wisconsin-Madison. Journal of Maps 5, 1-8.

w3schools, 2012. HTML5 Web Storage. Accessed: 7 May 2012.

http://www.w3schools.com/html/html5 webstorage.asp.

Westra, E., 2010. Python Geospatial Development. Packt Publishing, Birmingham. 


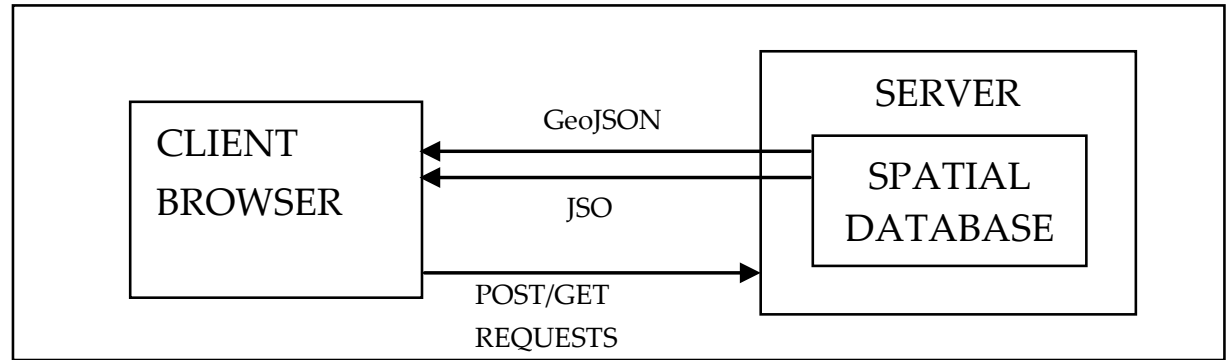

Figure 1: Basic interaction between client and server 


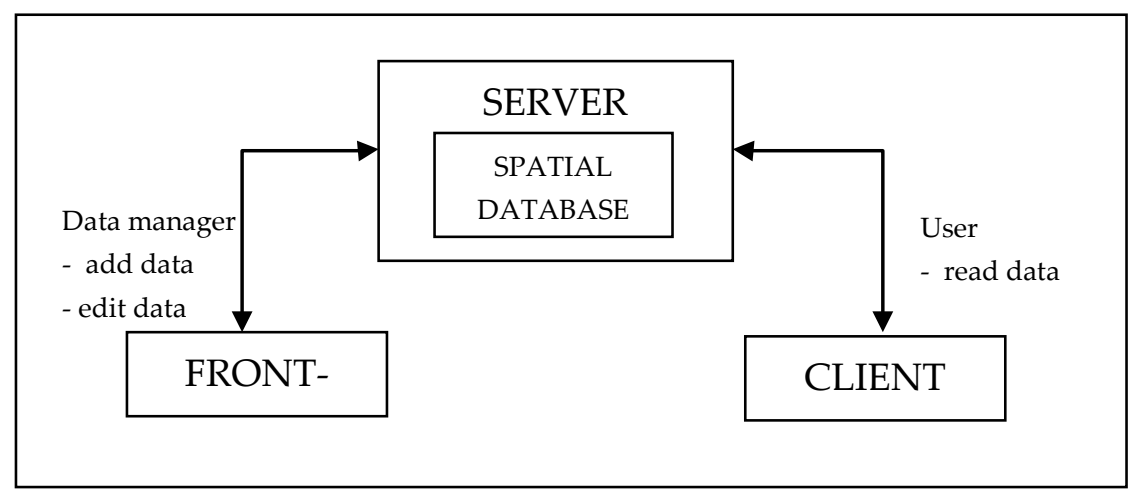

Figure 2: General system architecture 


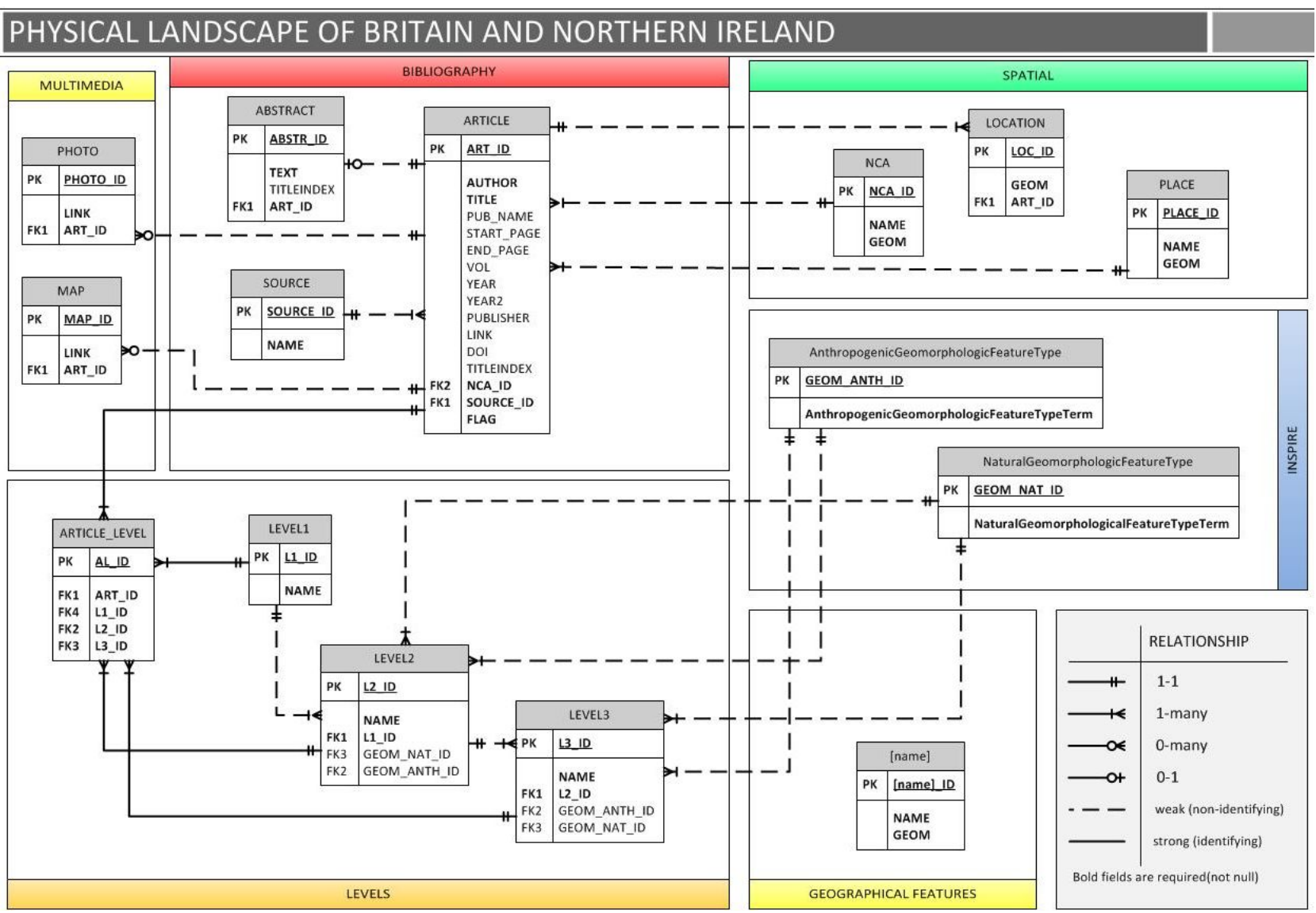

Figure 3: the database ER schema 


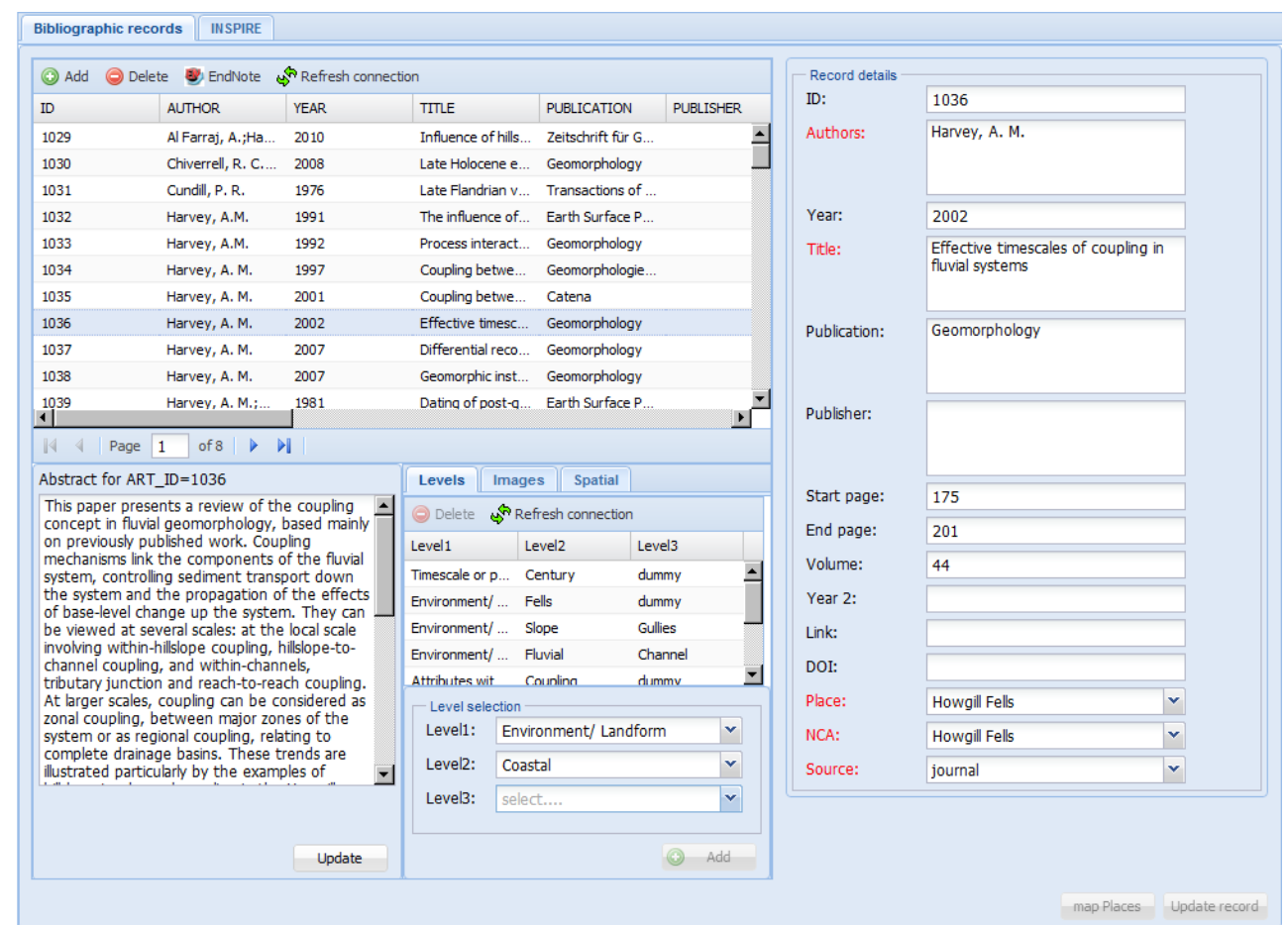

Figure 4: Database front-end (see Video 1 in Supplementary Materials) 


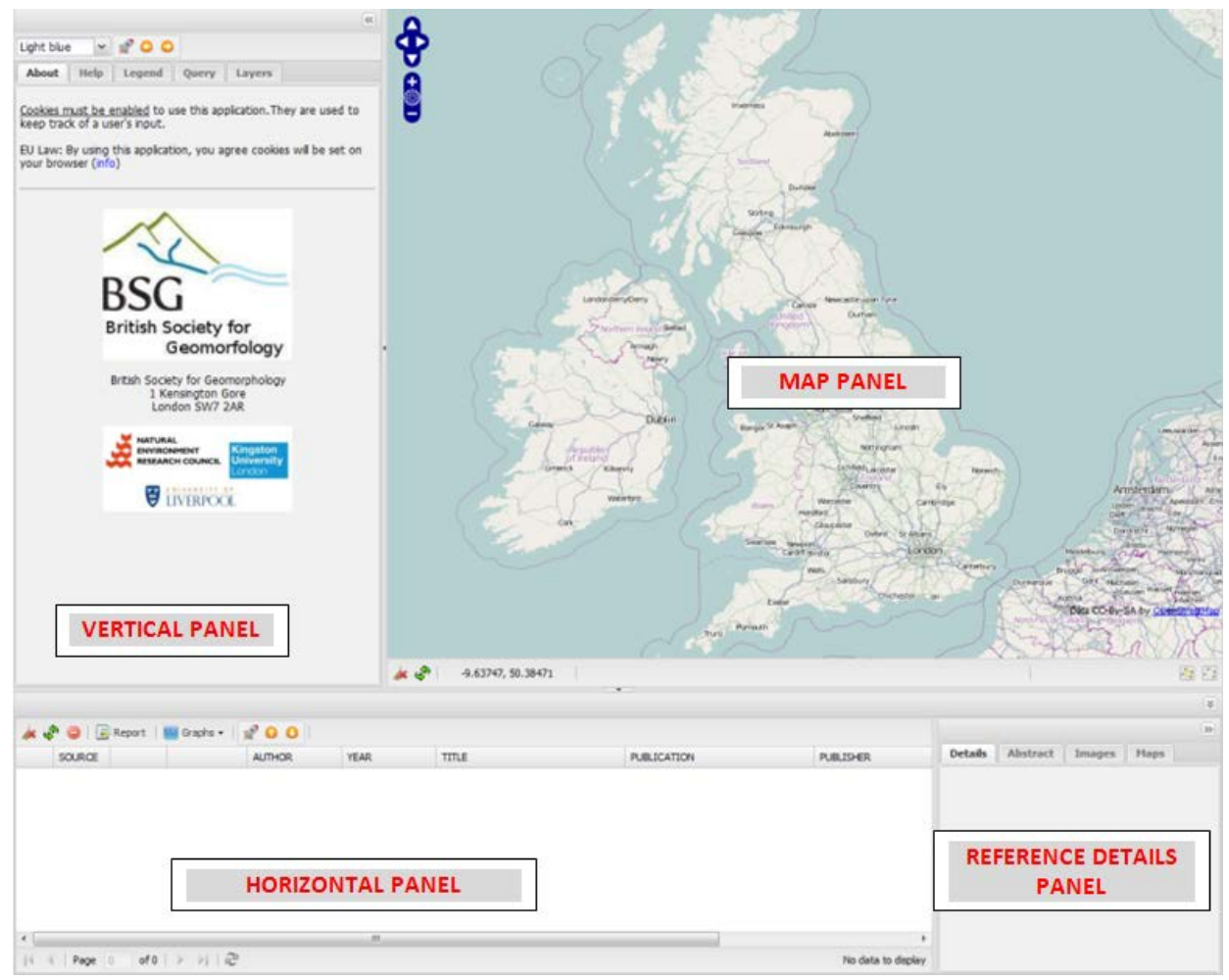

Figure 5: Web mapping application GUI (see Video 2 in Supplementary Materials) 


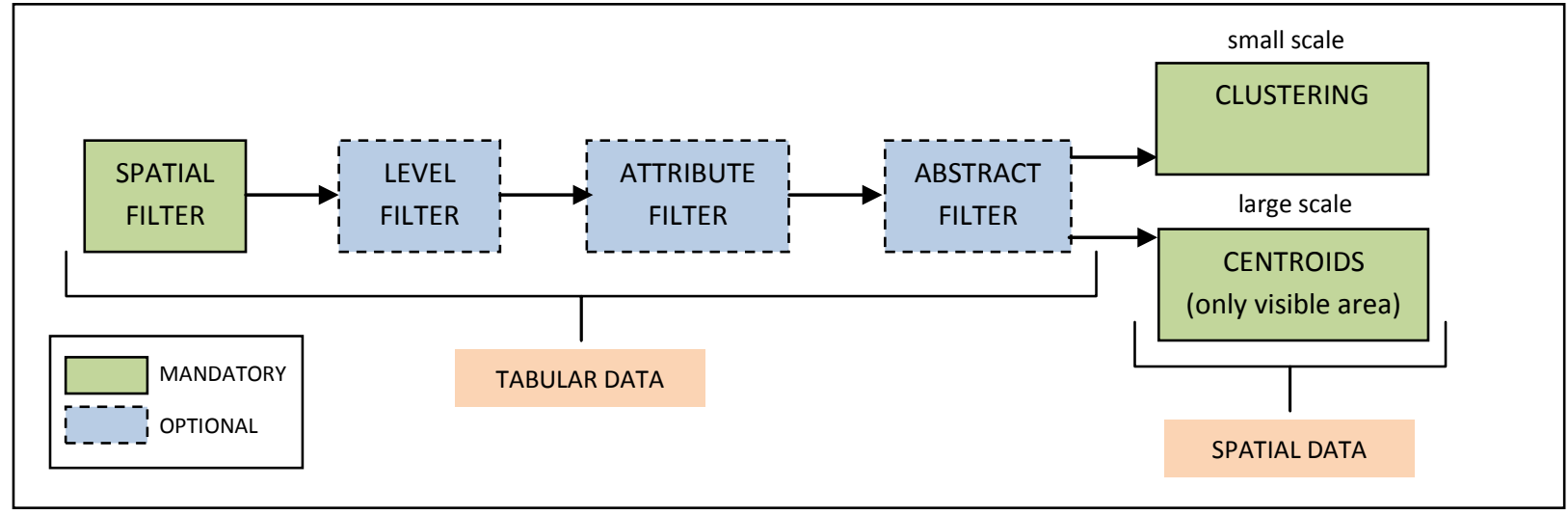

Figure 6: Building a query string 


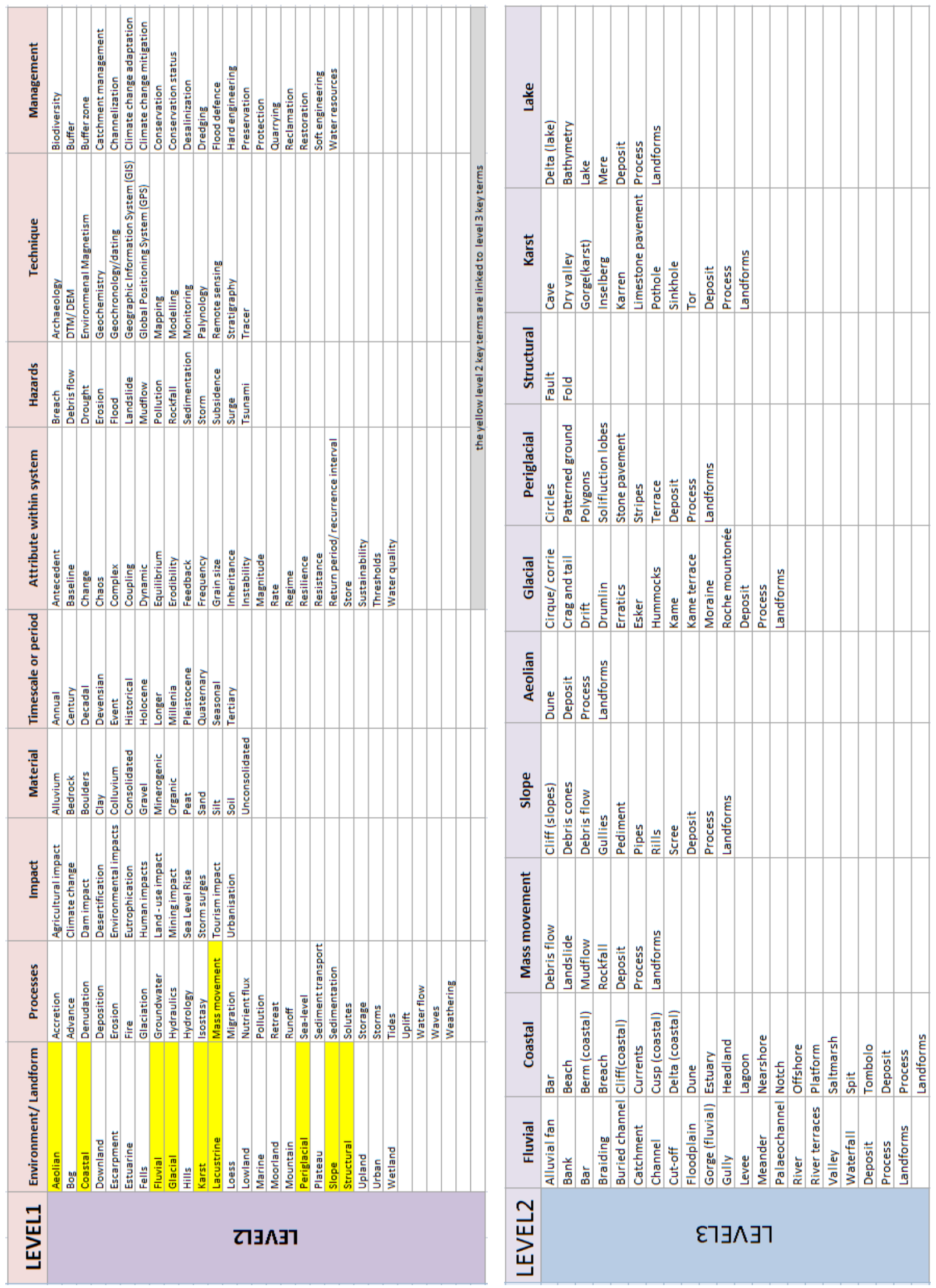

Table 1: Hierarchical 3-level key terms used to classify a reference. 